\title{
A Double H-Shaped Resonator for an Isotropic ENG Metamaterial
}

\author{
Jan Machac, Martin Rytir, Pavel Protiva, Jan Zehentner \\ Czech Technical University in Prague, \\ Technicka 2, 16627 Prague, Czech Republic \\ machac@fel.cvut.cz \\ Rytir.Martin@seznam.cz \\ protip1@fel.cvut.cz
}

\begin{abstract}
This paper presents a new planar particle showing negative effective permittivity under irradiation by an electromagnetic wave. The response of this particle, aimed to be sensitive to an electric field, is strongly anisotropic. The particle is used to design an isotropic epsilon-negative metamaterial in two forms. Firstly, a unit cell of the metamaterial consists of a cube bearing six proposed particles on its faces, located with specific orientations. The experiments showed that this unit cell is suitable for manufacturing an isotropic epsilon-negative metamaterial obtained by arranging these cells in a 3D cubic periodic system. The second form of an epsilon negative metamaterial with an isotropic response consists of the proposed planar particles themselves, distributed quasi-randomly, composing a 2D system and/or of particles placed in spherical shells and distributed fully randomly in a hosting material composing a 3D system. The isotropy of these systems was verified by measurements in a rectangular waveguide.
\end{abstract}

\section{INTRODUCTION}

Volumetric metamaterials (MTM) consist of a host medium in which insertions such as unit cells are located. Either a single specifically-shaped element, also known as a particle, or a suitably spatially-arranged set of particles forms the unit cell. The particles are anisotropic. Consequently, the medium composed by them generally also has an anisotropic response to an irradiating wave.

There are two ways leading to isotropy of an MTM. The first way utilizes a specific form of the unit cell satisfying symmetry of the selected crystallographic group and its periodical arrangement in space [1,2]. The second way is based on randomly located unit cells in the volume of the host [3].

The objective of this paper is to design and fabricate a volumetric isotropic epsilon negative (ENG) MTM applying a new kind of planar particle denoted as a double $\mathrm{H}$-shaped resonator (DHR). This particle is of a resonant nature, and consequently its operation is frequency selective. The interaction of the exciting field with the particle results in negative effective permittivity in a narrow frequency band above its resonant frequency. The layout of this particle is shown in Fig. 1a. The particle was designed and fabricated, and its response to an exciting electromagnetic wave is analyzed.
The behavior is compared with a particle that is already in use, composed of a planar dipole terminated by a loop inductor [2]. The six DHR particles are assembled to form a cube, as described in [1], to obtain an epsilon-negative cell with an isotropic response. These cells are fixed in space in a $3 \mathrm{D}$ rectangular periodical net to produce the volumetric isotropic MTM. The planar double H-shaped resonators were also tested to produce an ENG MTM with a quasi-2D isotropic response being randomly distributed in the R32 waveguide. The particles inserted into spherical plastic shells [3] and randomly poured into an R32 elevated rectangular waveguide were used to fabricate the real 3D volumetric ENG MTM with an isotropic response.

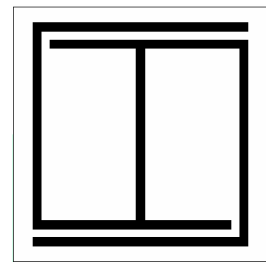

a

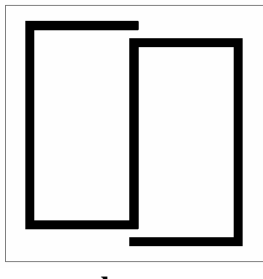

b

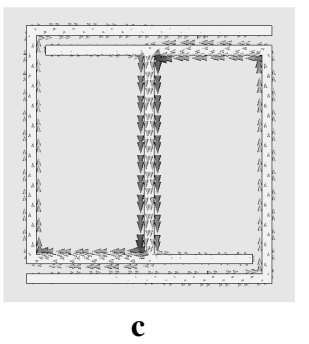

c
Fig. 1 Layout of an epsilon-negative particle - double $\mathrm{H}$-shaped resonator, the rear substrate side is without metallization (a), original rectangular-like resonator (b), current distribution on the DHR at resonance (c).

The particles themselves and the cubic cells were designed by the CST Microwave Studio, then fabricated and measured in the R32 waveguide with the propagating $\mathrm{TE}_{10}$ mode. The effective permittivity and permeability of a waveguide section with particles were calculated according to [4].

\section{Planar Double H-Shaped Resonator}

The aim of our work was to get the particle showing negative effective permittivity induced by the exciting electromagnetic wave. To do this, we have proposed a planar electric dipole terminated by a loop inductor in [2]. In its basic form, however, this particle is too large to be used for constructing a volumetric ENG MTM. Therefore, its form consisting of an inductor with two loops placed on two substrate sides was proposed [5]. The construction problem here is caused by the necessity to use via 
holes connecting the two inductor loops. Thus, our aim was to find a new planar particle able to resonate at a satisfactory low frequency that would therefore be compatible in size with our broad-side split ring resonators (BC-SRR) [3]. A solution was found after several iterations with a planar particle in the form of the folded dipole shown in Fig. 1b, which in this form represents a so-called rectangular-like resonator. The capacitive elements are here placed on the top and bottom dipole ends. Finally these capacitances were increased by extending an overlaying strip end and by adding an inside strip, and we obtained the double $\mathrm{H}$-shaped resonator, the layout of which is shown in Fig. 1a.

The DHR was optimized by the CST Microwave Studio using a substrate $0.2 \mathrm{~mm}$ in thickness, with permittivity 2.3 , loss factor 0.001 , and a metal cladding $17 \mu \mathrm{m}$ in thickness. The square substrate has finally its sides $6 \mathrm{~mm}$ in length, and the outer strip is $5.25 \mathrm{~mm}$ in length. The transmission characteristics $S_{21}$ of the DHR located in three positions at the middle of a TEM waveguide calculated by the CST Microwave Studio are plotted in Fig. 2. The resonant frequency is approximately $3.8 \mathrm{GHz}$. The ratio of the free space wavelength at the resonance and the particle size is about 13, so the MTM consisting of these particles can be assumed to be a homogeneous medium. Position 1 denotes the DHR perpendicular to the waveguide side walls and the central strip in the vertical position, so there is no interaction of the particle with the magnetic field in the TEM waveguide. The TEM waveguide cross section is the same as the substrate, i.e., $6 \times 6$ $\mathrm{mm}$. We have only one basic resonance at which the particle shows negative effective permittivity. At positions 2 and 3 the DHR is located parallel to the waveguide side walls with a vertical and horizontal position of the central strip, respectively. The magnetic field now interacts with the particle, and this causes the second magnetic resonance. As shown in Fig 3, this interaction results in negative effective permeability. This additional resonance is, however, well separated from the basic resonance aimed as the MTM working resonance. The DHR response is influenced by the position of the particle, and its behaviour is anisotropic. The calculated effective permittivity of the TEM waveguide section with the DHR located at its position 2 is shown in Fig. 3. The distribution of the electric current along the DHR strips located at position 1 and at the resonant frequency is shown in Fig. 1c.

The DHR was fabricated, see the inset of Fig. 4, on a ROGERS RT/duroid 5880 substrate with permittivity 2.22 and thickness $0.254 \mathrm{~mm}, \tan \delta=0.0012$ at $10 \mathrm{GHz}$ and metallization thickness $0.017 \mathrm{~mm}$. The outer strip is $5.25 \mathrm{~mm}$ in length and $0.2 \mathrm{~mm}$ in width, the same as the slot width. The particle is deposited on a substrate $6 \times 6 \mathrm{~mm}$ in area. The DHR was located in the R32 waveguide at the center of its cross-section in position 1. The corresponding measured transmission characteristic is plotted in Fig. 4. As expected, the response is weak, as we have only one tiny particle in the R32 waveguide of the rectangular cross section $72.14 \times 34.04 \mathrm{~mm}$. This fabricated particle was used in all the following experiments.

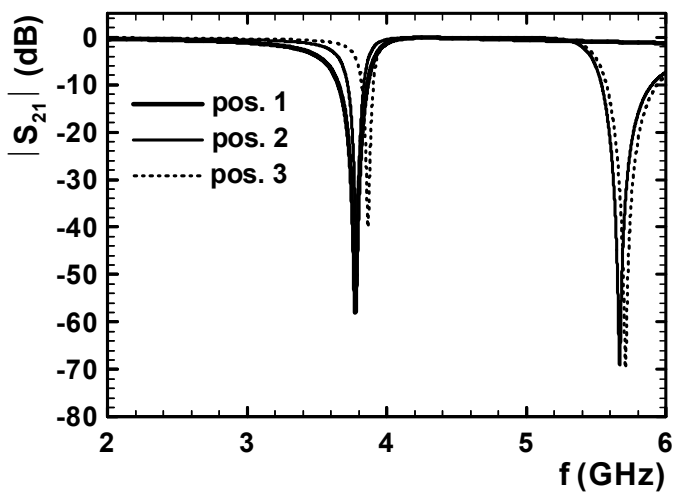

Fig. 2 Simulated transmission of the DHR located at the center of the TEM waveguide at the three positions defined in the text.

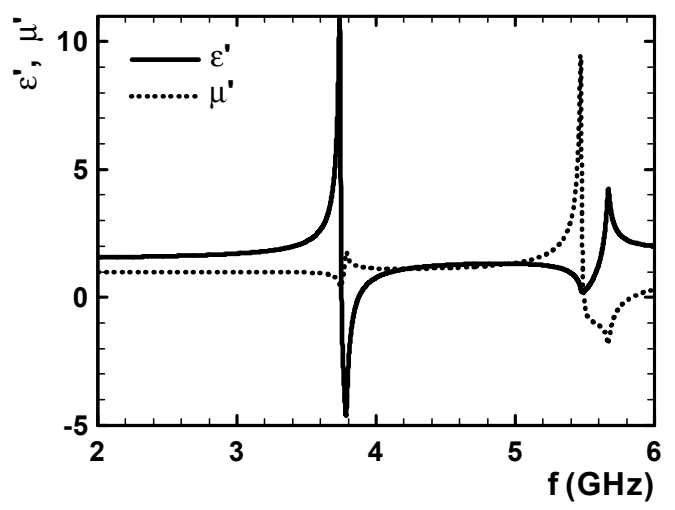

Fig. 3 Calculated [4] real parts of effective permittivity and permeability of the DHR located in the TEM waveguide in position 2.

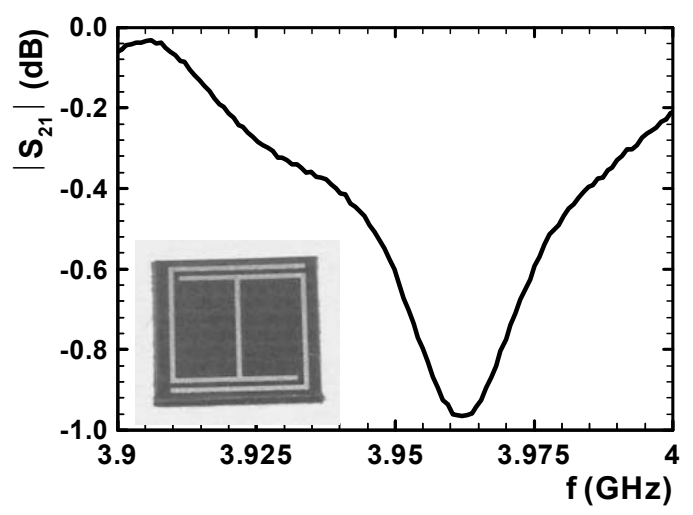

Fig. 4 Measured transmission of the DHR located at the center of the R32 waveguide. The inset shows the fabricated DHR.

\section{CUBe-LiKe Unit CeLl}

The DHRs were used to design the $3 \mathrm{D}$ isotropic unit cell which, irradiated by an electromagnetic wave, provides 
negative effective permittivity. This idea was carried into effect by placing the planar particles from Fig. 1a on the faces of a cube, creating a tetrahedral symmetrical system [1], see Fig. 5a. The interior of the cube is empty, since the particle substrates themselves form the faces of the cube. Fig. 5 shows three characteristic positions of the cube used in the experiments. We assume that the incident electric field is directed parallel to the $y$ axis, $z$ is the waveguide longitudinal axis, and the waveguide side walls are parallel to the $y z$ plane. Basic position 1 is shown in Fig. 5a, position 2 "on an edge" in Fig. 5b, and position 3 "on a node" in Fig. 5c. This unit cell was analyzed by the CST Microwave Studio only in the basic position and in the TEM waveguide. As in the case of a single DHR, the calculated transmission characteristic again shows two resonances, the lower resonance at about $4.08 \mathrm{GHz}$ providing negative effective permittivity in a frequency band tightly above it, and higher magnetic resonance at about $5.5 \mathrm{GHz}$ providing negative effective permeability tightly above it.

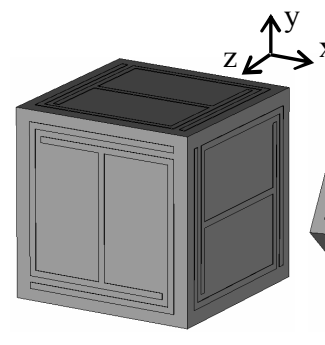

$\mathbf{a}$

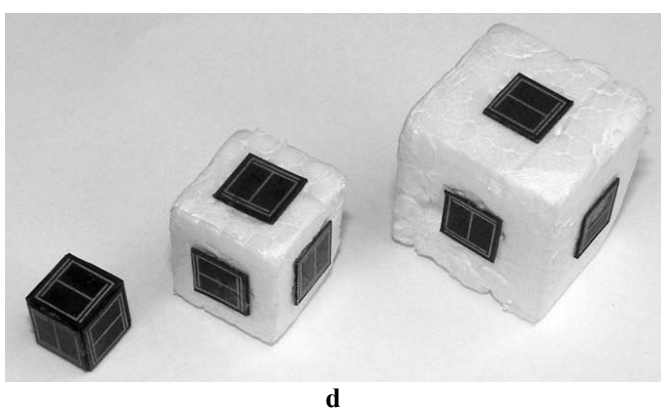

Fig. 5 A volumetric epsilon-negative cube-like cell composed of DHR, position 1 (a), position 2 (b), and position 3 (c). Fabricated cubes with 6x6, $12 \times 12$, and $15 \times 15 \mathrm{~mm}$ sides (d).

Three cube-like cells were fabricated by sticking the planar DHR particles on polystyrene cube faces $6 \times 6 \times 6,12 \times 12 \times 12$, and $15 \times 15 \times 15 \mathrm{~mm}$, see Fig. 5 d. The cubes composed of particular DHRs are complex systems with many internal couplings, which are more intensive at smaller dimensions. These structures were studied experimentally in the R32 waveguide. The presence of waveguide walls, the existence of the $\mathrm{TE}_{10}$ mode longitudinal magnetic field component and the nonhomogeneity of this field influence the electromagnetic response of the cell inserted in the waveguide. These effects result in different responses of the cubes in comparison with the responses of the single planar particles. The transmission of the R32 waveguide with the cube-like unit cell from Fig. 5d with dimensions $15 \times 15 \times 15 \mathrm{~mm}$ measured when located in the waveguide center in the three positions, as defined above, is shown in Fig. 6. This transmission depends only slightly on the selected position of the cube. We can therefore conclude that this cube-like unit cell has an isotropic response and is a suitable building block for an isotropic ENG MTM, and is in fact the most suitable from those shown in Fig. 5d. This MTM is obtained by assembling these cells in a $3 \mathrm{D}$ cubic periodic net.

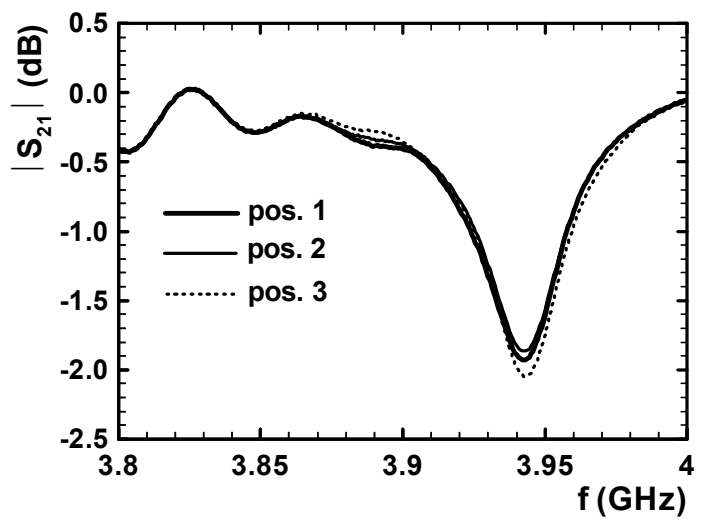

Fig. 6 Measured transmissions of the R32 waveguide with a cube with dimensions $15 \times 15 \times 15 \mathrm{~mm}$ assembled from six DHRs for its different positions.

\section{2D QUASI ISOTROPIC ENG MTM COMPOSED OF DHRS}

The 2D quasi random ENG MTM was prepared as a polystyrene parallelepiped $50 \times 50 \mathrm{~mm}$ in area cut into three slices that are equal in height of $10 \mathrm{~mm}$. An equal number of DHRs is inserted periodically in the nodes of a squared net with random orientation in each slice, see inset of Fig. 7. Consequently, by changing the mutual orientations of the slices in the parallelepiped by 90-degree rotations we obtained 64 different parallelepipeds with randomly distributed particles inside, assuming that the vertical order of the slices is kept. For the isotropy test the stacks of these three slices were inserted into the R32 rectangular waveguide in such a way that the two sides were parallel to the field propagation. If the parallelepiped is isotropic and the geometry of the setup is unchanged, the measured scattering parameters should remain invariant for each 90-degree rotation of the parallelepiped around its central vertical axis. The arithmetic mean value of 64 times measured $S_{21}$ is plotted in Fig. 7, together with its disperse. This disperse is very small, so the MTM sample can be viewed as a 2D isotropic medium. The more particles there are, the wider is the stop-band in which the MTM shows negative effective permittivity. 


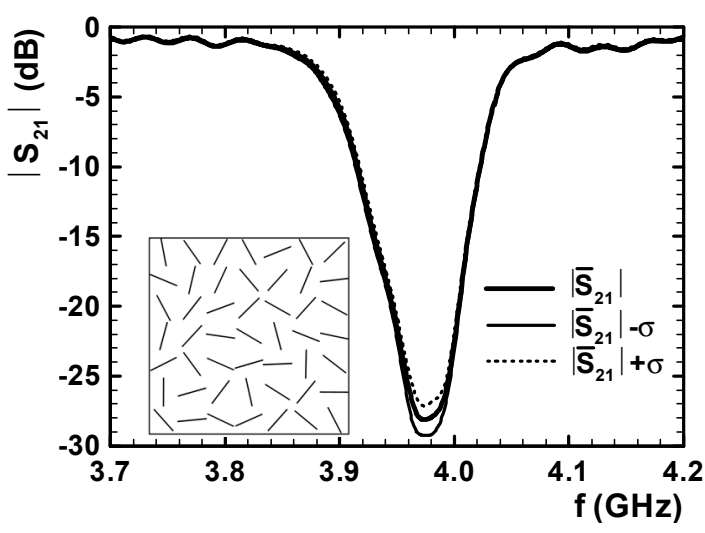

Fig. 7 Arithmetic mean value of the transmission through 64 parallelepipeds of the 2D ENG MTM in the R32 waveguide with 147 DHRs and its disperse. The inset shows the top view of the particles located in one slice.

\section{3D ISOTROPIC ENG MTM COMPOSED OF DHRS}

The actual isotropic 3D mu negative MTM with really random location of the unit cells was achieved by BC-SRRs boxed in polystyrene spherical shells filling up the volume of a cube with edges $72 \mathrm{~mm}$ in length, as we presented in [3]. The same technology was adopted here to fabricate a 3D isotropic ENG MTM using the DHRs. The inset of Fig. 8 shows a disassembled shell together with the particle. The metamaterial specimen consisting of 256 shells with the DHRs was repeatedly measured in a raised R32 rectangular waveguide, changing the position of the shells each time. The arithmetic mean value of these measurements, together with their disperse, are plotted in Fig. 8. This disperse is now bigger than in the case of a 2D MTM, but it is still comparable with that obtained in [3] for the MTM consisting of BC-SRR. Consequently, Fig. 8 shows that the studied ENG MTM consisting of DHRs boxed in the plastic spherical shells behaves as an isotropic metamaterial.

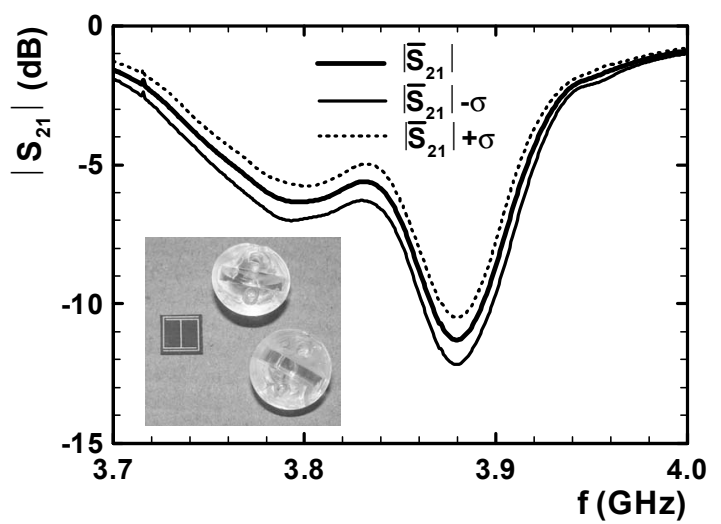

Fig. 8 Arithmetic mean value and its disperse of 25 measurements of transmission through the 3D ENG MTM in the raised R32 waveguide with 256 DHRs placed in spherical shells and randomly located in the volume of the cube with sides $72 \mathrm{~mm}$ in length.

\section{CONCLUSIONS}

This paper presents a new form of a double $\mathrm{H}$-shaped planar resonator aimed to be primarily sensitive to the electric field of an incident electromagnetic wave. Due to intensive resonance, this particle excites negative effective permittivity. This DHR particle is small enough, and is consequently ready for application in isotropic metamaterials. There is no need to use vias, as in the case of a reduced size standard electric dipole terminated by a two loop conductor [5]. Production of the new particle is simple and, therefore, cheap.

The double H-shaped resonator was optimized by the CST Microwave Studio. The simulations and experiments confirm its behavior. Cubic-like unit cells bearing on their faces six identical DHRs with proper symmetry were proved to be suitable building blocks for a bulk isotropic ENG MTM consisting of a cubic periodic system of these cells. The concept of manufacturing bulk isotropic MTMs, both 2D with quasi-randomly distributed anisotropic particles and 3D with fully randomly distributed anisotropic particles, has also been proved practicable in the case of DHRs. In the 3D case, the DHRs were boxed in polystyrene spherical shells. The frequency band of composites with randomly located unit cells is wider than the band of a single particle, and at the same time the response is more intensive.

\section{ACKNOWLEDGEMENT}

This work has been supported by the Grant Agency of the Czech Republic under project 102/06/1106 "Metamaterials, nanostructures and their applications".

\section{REFERENCES}

[1] J. D. Baena, L. Jelinek, R. Marques and J. Zehentner, "Electrically Small Isotropic Three-Dimensional Magnetic Resonators for Metamaterial Design”, Applied Phys. Lett, Vol. 88, 134108, 2006.

[2] J. Machac, P. Protiva, J. Zehentner, "Isotropic Epsilon-Negative Particles," 2007 IEEE MTT-S Int. Microwave Symp. Dig., Honolulu, USA TH4D-03, June 2007.

[3] L. Jelinek, J. Machac, J. Zehentner, "A Magnetic Metamaterial Composed of Randomly Oriented SRRs”, Proceedings PIERS 2007, Beijing 2007, China, pp. 474-477.

[4] D. R. Smith, S. Schultz, P. Markos, C. S. Soukoulis: "Determination of effective permittivity and permeability of metamaterial from reflection and transmission coefficients", Phys. Rev. B, Vol 65, 195104, 2002.

[5] P. Protiva, J. Machac, J. Zehentner, "Particle for an Isotropic Metamaterial with Negative Permittivity", EMTS 2007 - International URSI Commission B Electromagnetic Theory Symposium, July 2007, Ottawa, Canada, CD-ROM. 